\title{
RELATIVISTIC REFERENCE FRAMES OF LOCAL OBSERVER AND SPACE RADIOINTERFEROMETER
}

\author{
A.N. ALEXANDROV, S.L. PARNOVSKY, V.I. ZHDANOV \\ Astronomical Observatory of Kiev University \\ Observatorna St. 3 \\ 252053 Kiev, USSR
}

In a considerable number of works on relativistic astrometry (see, e.g. Kovalevsky and Brumberg 1986) the reference frames (RFs) are introduced either by means of coordinate representation of a space-time metric, such as using harmonicity conditions (Brumberg and Kopejkin 1989), or on the basis of invariant constructions like Fermi coordinates (Synge 1960; Ashby and Bertotti 1986; Boucher 1986). Both approaches must, probably, be combined in applications. We consider the local observer RFs (LORFs) based on the Fermi coordinates and on the optical ones (Synge 1960), which are rigorously defined for a general metric and are directly related to observable quantities. In particular, the optical RF operates with the observed direction of the light source, whereas the Fermi RF seems to be a natural generalization of the classical Cartesian RF.

The specificity of the radiointerferometry in space leads to the following questions dealt with in this report.:

(i) In view of a large baseline $\left(10^{5}\right.$ to $\left.10^{6} \mathrm{~km}\right)$ of the space radiointerferometer (SRI) a more accurate account of relativistic effects in calculation of the delay time $\tau$ is needed in comparison with VLBI on the Earth. As in Finkelstein et al. (1983) and in Zeller et al. (1986), we are guided by accuracy of $1 \mathrm{ps;}$, however, for the above baseline size this requires the relative accuracy of $10^{-12}$, that is two orders higher than that discussed by Finkelstein $e t$ al. and by Zeller $e t$ al.

(ii) In construction of LORFs in the nearby space taking into account of the Earth-Moon gravitational field is desirable. This is not allowed by the methods of Ashby and Bertotti (1985) and Boucher (1986) where the Fermi RF in a fictitious background metric has been treated by using the Taylor expansion in spatial coordinates. We used the other way to construct the transformation to the Fermi and optical RFs, which embraces the case when the distance from gravitating bodies is comparable with the SRI base. The transformations are obtained according to the definitions of Synge (1960) by means of direct solution of the geodesic equations in the first weak-field approximation. If one rules out the Earth-Moon field, then in the case of geocentrical Fermi RF the transformations can be reduced to the results of Ashby and Bertotti (1985) and Boucher (1986). 
On the basis of these results we obtain the relation for $\tau$ in the LORF, in which at the limit of the accuracy there is the input of the geodesic precession of the LORF for the measurement time of $10^{3}$ $\mathrm{sec}$, and of the homogeneous part of the solar gravitational field. For VLBI on the Earth (relative accuracy $10^{-10}$ ) the relation for $\tau$ in the Fermi RF agrees with the result of Zhu and Groten (1988) and differs from that of Finkelstein et al. (1983) and Zeller $e$ al . (1986) by the scale factor $\left(1-\varphi_{0}\right)$ where $\varphi_{\mathrm{o}}$ is the solar gravitational potential. The reason for the discrepancy is that another normalization of the distance was employed in Finkelstein et al. (1983) and Zeller et al. (1986).

For SRI with a large baseline the calculation of the gravitational input $\tau_{\text {erav }}$ in $\tau$ is somewhat different from that of Finkelstein et al. (1983). Apart from the Sun and the Earth, the gravitational contributions of Jupiter and Saturn are essential, the inputs of the other planets are essential only for the received signals passing in the close neighborhood of the planets. In analogous cases, (a) the postpost-Newtonian corrections to the solar gravitational field and (b) angular momentum of the Sun give also the input of some ps. The input (b) linear in $\mathrm{J}$ is derived in harmonic coordinates, the PPN contribution (a) is given by the results of Brumberg (1987).

With the aim of rigorous formulation of some LORF problems, we have considered the methods of construction of the Fermi and optical RFs using the exponential mapping technique (EMT) in curved space-times. It gives a consistent description of extended relativistic systems in the closed form, including the cases when their sizes are comparable with the characteristic scale of the gravitational field. EMT allows one to specify an even $\mathrm{P}$ by means of a four-vector $\mathrm{y}$ at the position of the observer, the components of $y$ being Riemannian normal coordinates of $P$. The algorithms to find all physical characteristics in this coordinate system through observables are known (Alexandrov 1981). To apply these to the above LORFs one must take into account the variation of the mapping $\mathrm{P} \rightarrow \mathrm{y}$ under the shift along the observer's trajectory and perform (3+1)-splitting of the space-time. This is described by Jacobi fields which satisfy the geodesic deviation equation. By means of EMT we have obtained the transformation of observables to the LORFs and the exact equations of the test body motion in the LORFs. The resulting expressions are presented in the covariant form and may be computed either by means of covariant Taylor expansion or in the weakfield approximation.

\section{References}

Kovalevsky, J. and Brumberg, V.A.: 1986, Relativity in Celestial Mechanics and Astrometry, Proc. IAU Symp. No. 114, D. Reidel, Dordrecht.

Brumberg, V.A. and Kopejkin, S.M.: 1989, "Relativistic theory of celestial reference frames", in J. Kovalevsky, I.I. Mueller, B. Kolaczek (eds.) Reference Frames in Astronomy and Geophysics , Kluwer Academic Publishers, Dordrecht, pp. 115-141.

Synge, J.L.: 1960, Relativity: The General Theory, North-Holland, Amsterdam

Ashby, N., and Bertotti, B.: 1986, Phys. Rev. D 34, 2246.

Boucher, C.: 1986, "Relativistic effects in geodynamics", in J. Kovalevsky and V.A. Brumberg (eds.) Relativity in Celestial Mechanics and Astrometry, D. Reidel, Dordrecht, pp. 241-253.

Finkelstein, A.M., Kreinovich. V.Ya., and Pandey, S.N.: 1983, Ap. Sp. Sci. 94, 233.

Zeller, G., Soffel, M. Ruder, H., and Schneider, M.: 1986, Veröff. d. Bayer Komm. Int. Erdmes. Munich 48, 218.

Zhu, S.Y., and Groten, E.: 1988, Manuscr. Geod. 13, 33. 
Brumberg, V.A.: 1987, Кинемат. и физ. небес. тел 3, 8.

Alexandrov, A.N.: 1981, Acta Phys. Polon. B12, 523.

\section{Discussion}

KOPEJKIN: I want to point out that the method of construction of local coordinate systems in the vicinity of a massive body was developed by Fukushima et al. as well.

ZHDANOv: The difference of our consideration from other works is that we avoid expansion in spatial coordinates and therefore we do not need to separate the gravitational field of the Earth in construction of the LORFs.

KLIONER: I think that to derive the relativistic correction due to the rotation of the bodies and in particular due to the rotation of the Sun, you had to obtain the trajectory of the light considering the bodies' rotation. Did you really obtain this trajectory and, if yes, what kind of coordinates (i.e. cartesian or spherical) did you use?

ZHDANOv: We actually have obtained this trajectory in the cartesian coordinates. 